\title{
In Situ Detection of Protein Complexes and Modifications by Chemical Ligation Proximity Assay
}

\author{
Rui Hong*, Esteban Roberts and Christopher Bieniarz \\ Technology and Applied Research, Ventana Medical Systems, Inc. \\ 1910 E. Innovation Park Drive, Tucson, AZ 85755, United States
}

\section{Supporting Information}

\section{Table of Contents}

1. Structures of reagents used for antibody modification

2. Synthetic scheme for Conjugate A (Figure S1)

3. Supplemental Data

3.1. Typical SEC chromatograph of purification of Conjugate A (Figure S2)

3.2. Detection of HA-tag as a hapten (Figure S3)

3.3. Activity of Acetylene for Click reaction in Conjugate B (Figure S4)

3.4. Determine conditions for complete cleavage of HA-tag from antibody (Figure S5)

3.5. Preservation of haptenated antibody under the reducing conditions (Figure S6)

3.6. Detection of E-cad/p120 in T47D and MDA-MB-453 cells by PLA and CLiPA (Figure S7)

3.7. Control experiment of CLiPA for pEGFR detection (Figure S8) 


\section{Structures of reagents used for antibody modification}

- SPDP-dPEG8-NHS ester was purchased from Quanta BioDesign, Ltd. (Plain City, OH, USA)

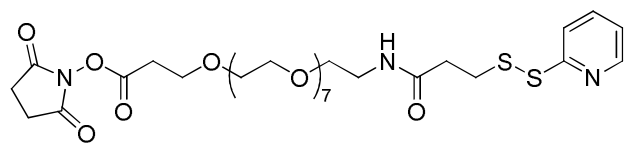

- Peptide Cys-HA-N3 was obtained from Bio-Synthesis, Inc (Lewisville, TX, USA)

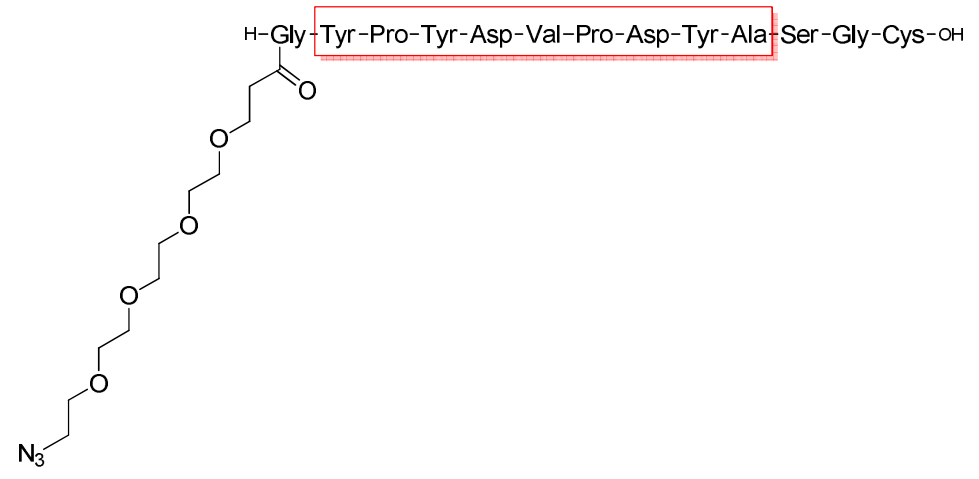

- Alkyne-PEG4-NHS ester from Click Chemistry Tools (Scottsdale, AZ, USA)<smiles>C=CCOCCOCCOCCOCCC(=O)ON1C(=O)CCC1=O</smiles> 


\section{Synthetic scheme for Conjugate A}

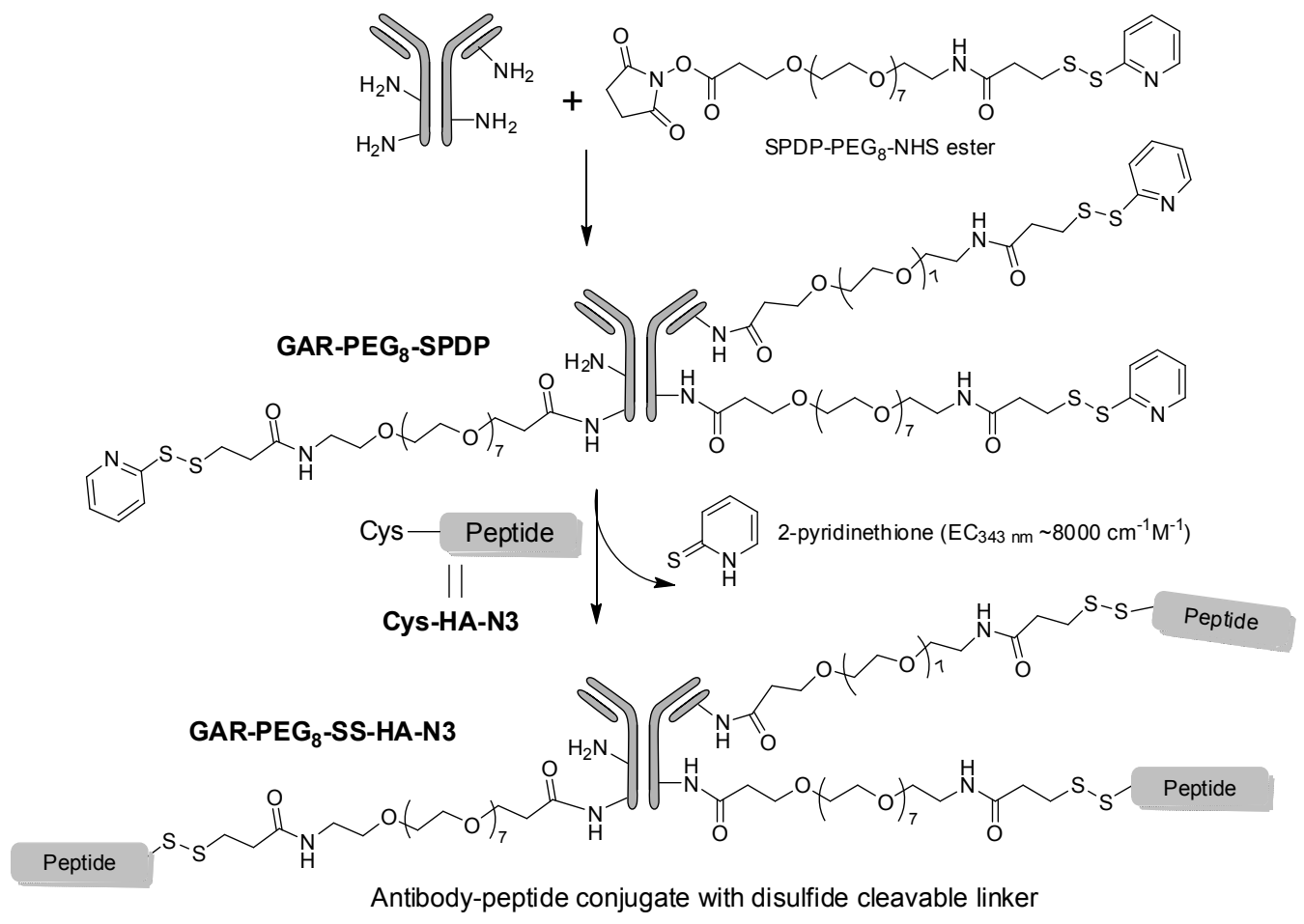

Figure S1. Synthetic scheme for GAR-P ${ }_{8}-S S-H A-N_{3}$ (Conjugate A)

\section{Supplemental Data}

\subsection{Typical SEC chromatograph of purification of Conjugate A}

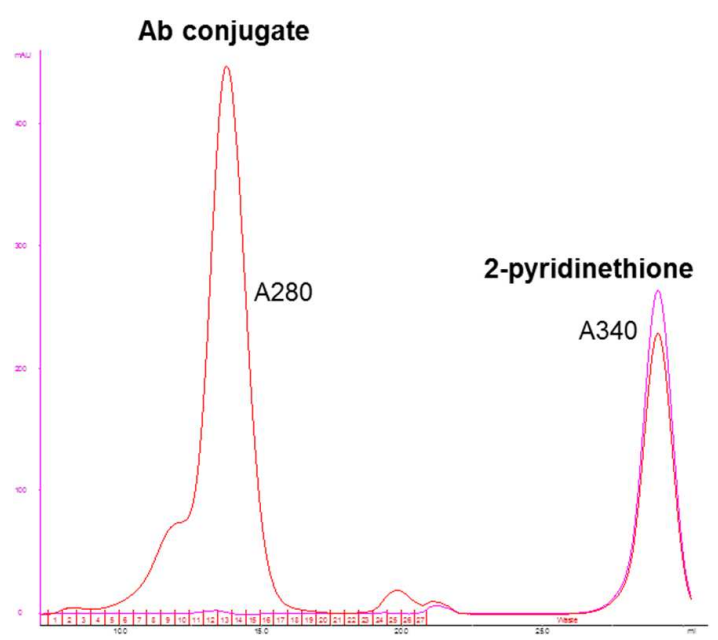

Figure S2. A typical SEC chromatogram for purification of the GAR-HA-tag peptide conjugate (Conjugate A). 
3.2. Detection of HA-tag as a hapten

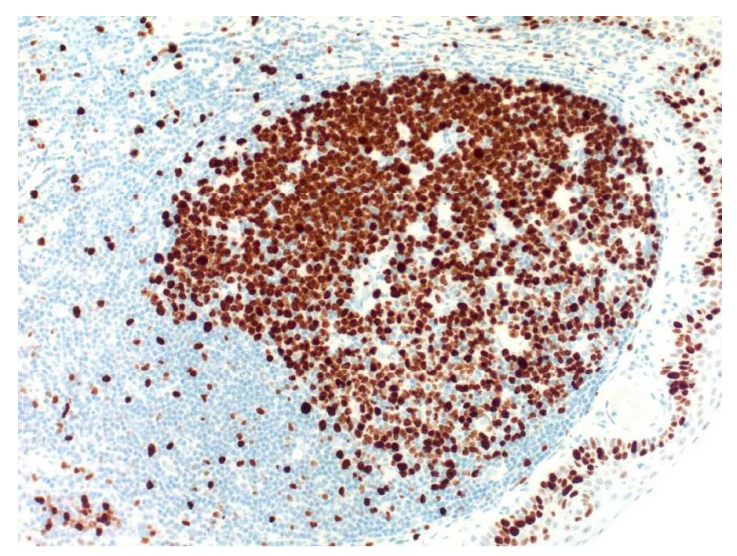

Figure S3. Detection of Ki67 protein in FFPE tonsil tissue by HA-tag using IHC and DAB chromogenic detection system.

\subsection{Activity of Acetylene for Click reaction in Conjugate B}

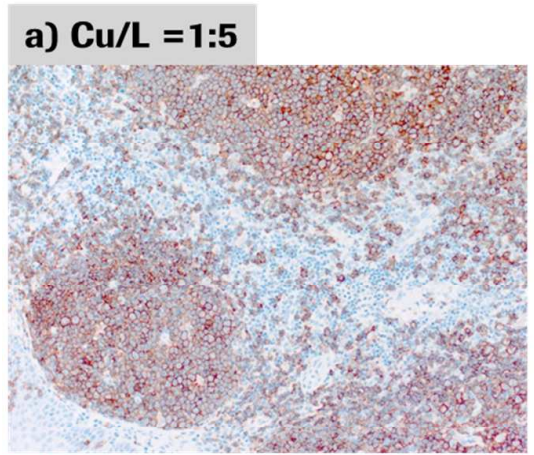

$$
\text { b) } \mathrm{Cu} / \mathrm{L}=1: 3
$$
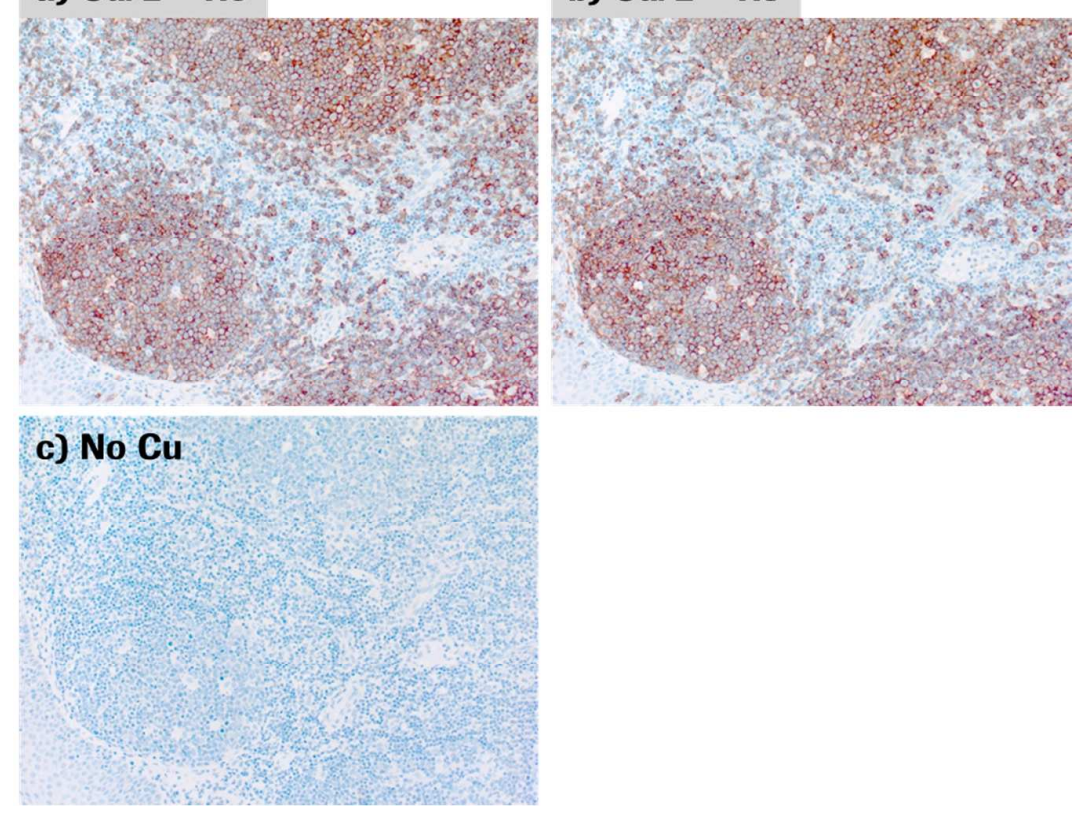

Figure S4. Detection of CD20 in tonsil sections using GAM-PEG4-CCH conjugate: (a) $\mathrm{Cu}(\mathrm{I}) / \mathrm{THPTA}$ ratio of $1: 5$, (b) $\mathrm{Cu}(\mathrm{I}) / \mathrm{THPTA}$ ratio of $1: 3$, and $(\mathrm{C})$ negative control with no $\mathrm{Cu}(\mathrm{I})$ added. 


\subsection{Determine conditions for complete cleavage of HA-tag from antibody}

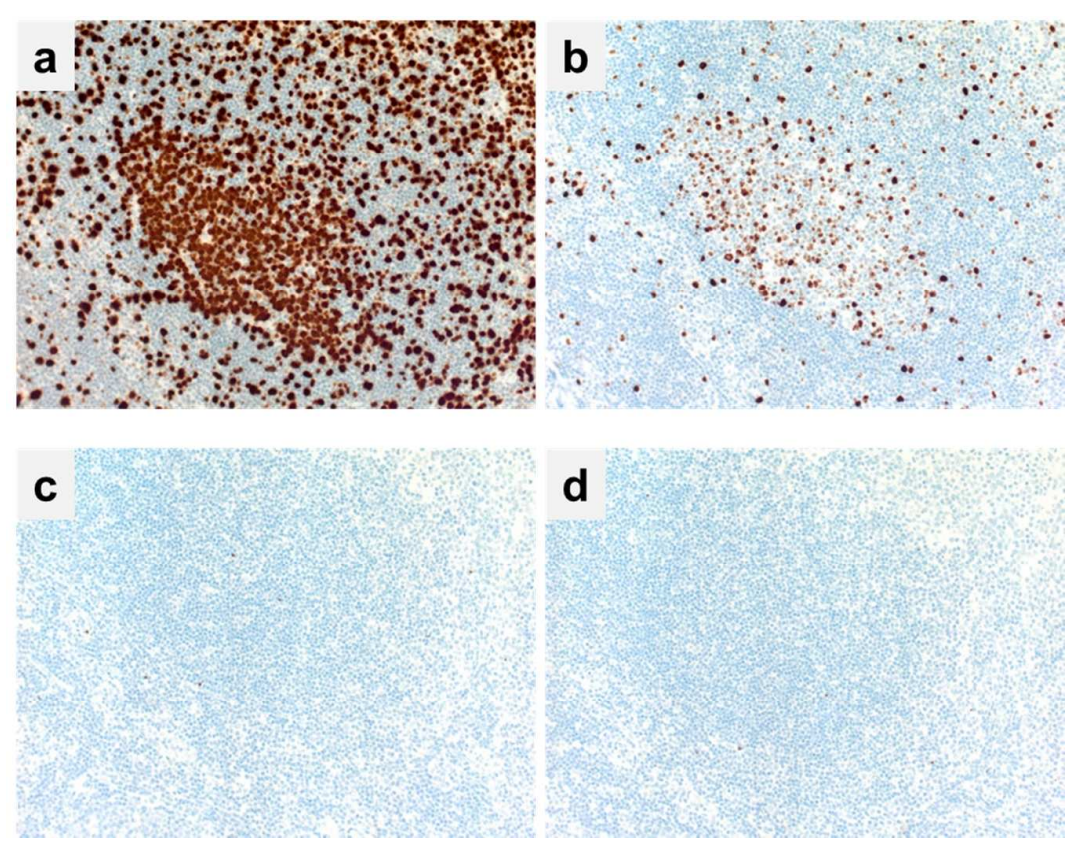

Figure S5. Determination of DTT concentrations to achieve complete cleavage of HA-tag: (a) no DTT, (b) $50 \mathrm{mM}$ DTT, (c) $75 \mathrm{mM} \mathrm{DTT}$, and (d) $100 \mathrm{mM} \mathrm{DTT} \mathrm{added.}$

\subsection{Preservation of haptenated antibody under the reducing conditions}

We used VENTANA OptiView DAB IHC detection kit for the IHC detection of protein bcl-6 in FFPE tonsil tissues to demonstrate that antibody is unaffected under the reducing conditions for cleaving the hapten in CLiPA. Specifically, mouse-anti-bcl6 (Ventana 7604241) was use as the primary antibody followed by adding OptiView HQ Universal Linker which contains hapten HQ labeled secondary antibodies. The reducing reagent $(100 \mathrm{uL}$ per slide) was then added under the same conditions as in CLiPA (the effective concentration of TCEP is 1/3 to 1/4 of the indicated concentration since there is about 200-300 uL liquid existing on the slide). After which, OptiView HRP Multimer was added followed by DAB chromogenic detection. As show in Figure S6, there is no visible difference between PBS and TCEP treated slides, indicating that the reducing conditions in CLiPA is not causing the dissociation of the bound hapten. In addition, it has been established that the IgG structure is maintained both by disulfide bonds and by noncovalent interactions between the heavy chains and light-heavy chains. (Wang, W., et al. (2006) Antibody structure, instability, and formulation J. Pharm. Sci. 96, 1-26). 

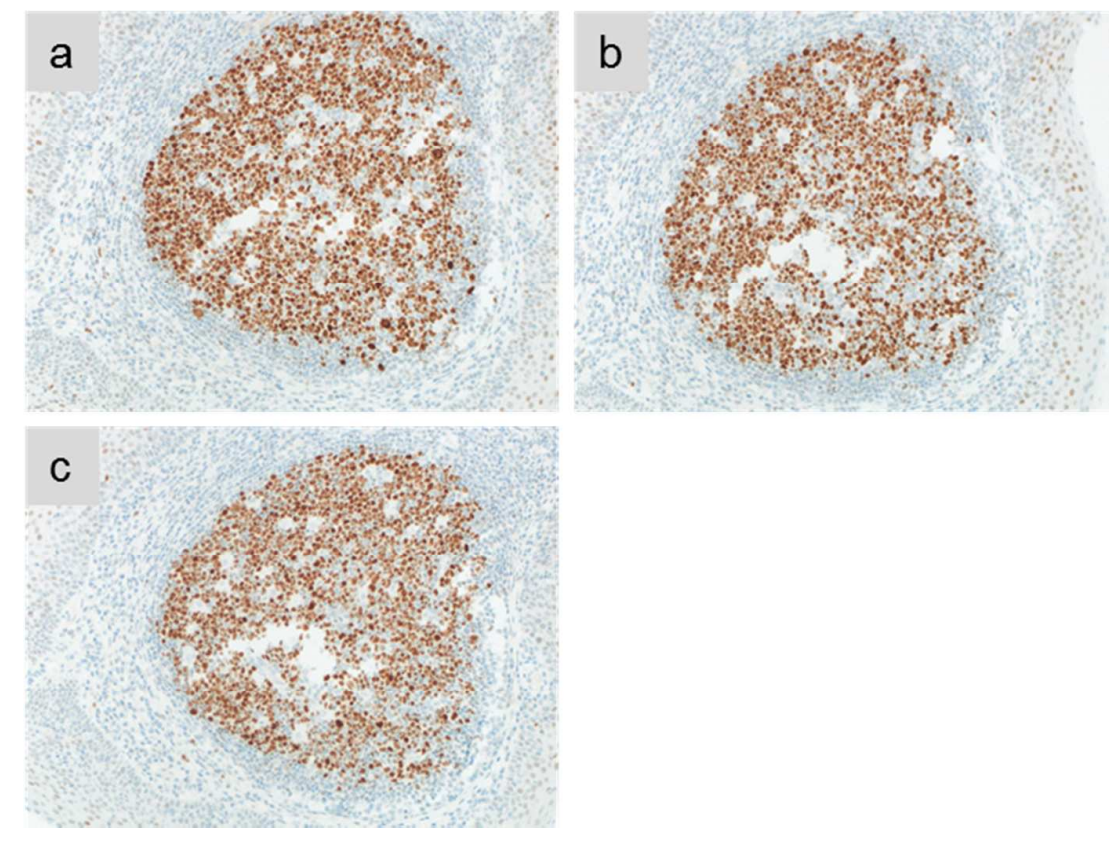

Figure S6. Detection of bcl-6 in FFPE tonsil with different concentrations of TCEP (a) PBS control, (b) 50mM TCEP and (c) $100 \mathrm{mM}$ TCEP.

\subsection{Detection of E-cad/p120 in T47D in MDA-MB-453 cells by PLA and CLiPA}
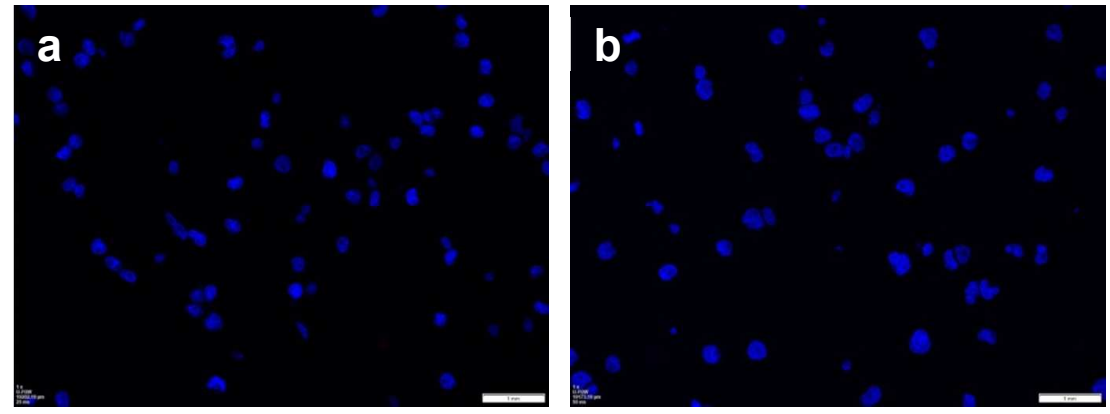

Figure S7. Detection of E-cad/p120 in T47D in MDA-MB-453 cells by (a) PLA, and (b) CLiPA. 


\subsection{Control experiment of CLiPA for pEGFR detection}

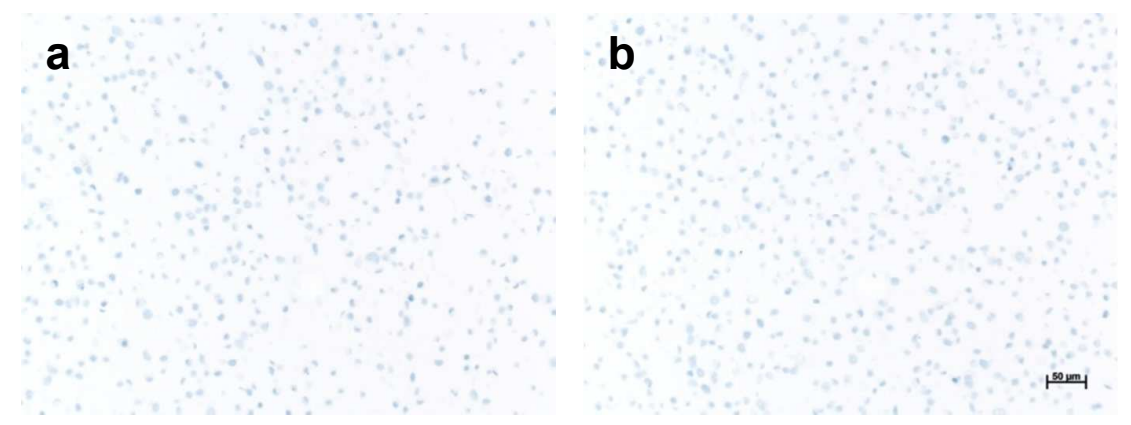

Figure S8. CLiPA with omission of chelated Cu(I) catalyst for phosphor EGFR detection in SKBr3 cells (a) $+E F G$, and (b) $-E G F$. 